\title{
A Finite Element Method for First-Order Hyperbolic Systems
}

\author{
By Mitchell Luskin*
}

\begin{abstract}
A new finite element method is proposed for the numerical solution of a class of initial-boundary value problems for first-order hyperbolic systems in one space dimension. An application of our procedure to a system modeling gas flow in a pipe is discussed. Asymptotic error estimates are derived in the $L^{2}$ norm in space.
\end{abstract}

1. Introduction. We propose and analyze a new finite element method for the numerical solution of a class of initial-boundary value problems for first-order hyperbolic systems in one space dimension. Our method is based on a procedure given by Platzman [9]. A generalization of our procedure for problems in two space dimensions will be treated in a later paper [8].

We consider problems in one space dimension of the form

$$
\begin{aligned}
& u_{t}+\left(a_{12}(x, t) v\right)_{x}=f_{1}(x, t, u, v), \quad(x, t) \in[0,1] \times[0, T], \\
& v_{t}+a_{21}(x, t, u, v) u_{x}+a_{22}(x, t, u, v) v_{x}=f_{2}(x, t, u, v),
\end{aligned}
$$

$$
(x, t) \in[0,1] \times[0, T],
$$

$$
\begin{array}{lll}
u(x, 0)=u_{0}(x), & v(x, 0)=v_{0}(x), & x \in[0,1], \\
v(0, t)=g_{0}(t), & v(1, t)=g_{1}(t), & t \in[0, T] .
\end{array}
$$

We assume that $(u(x, t), v(x, t))$ is a smooth solution of $(1.1)$. Let $A$ be a compact neighborhood in $[0,1] \times[0, T] \times \mathbf{R} \times \mathbf{R}$ of the set

$$
\{(x, t, u(x, t), v(x, t)) \mid(x, t) \in[0,1] \times[0, T]\},
$$

and assume that there exists a positive constant, $\alpha$, such that

$$
a_{12}(x, t) \geqslant \alpha, \quad a_{21}\left(x, t, s_{1}, s_{2}\right) \geqslant \alpha,
$$

for all $\left(x, t, s_{1}, s_{2}\right) \in A$. We assume also that $a_{i j}\left(x, t, s_{1}, s_{2}\right)$ and $f_{i}\left(x, t, s_{1}, s_{2}\right)$ are Lipschitz continuous functions of their arguments for $\left(x, t, s_{1}, s_{2}\right) \in A$.

Initial-boundary value problems of the form (1.1) occur, for example, by scaling the space variable, $x$, of the following first-order system modeling the transient behavior of isothermal gas flowing in a pipe:

Received August 2, 1978; revised January 15, 1980.

AMS (MOS) subject classifications (1970). Primary 65N30, 65M15.

*Supported by AFOSR under Contract F49620-79-C-0149 and by a Faculty Research Fellowship from the Horace Rackham School of Graduate Studies, The University of Michigan. 


$$
\begin{aligned}
& \rho_{t}+G_{x}=0, \quad(x, t) \in[0, L] \times[0, T], \\
& G_{t}+\left(\sigma^{2}-G^{2} \rho^{-2}\right) \rho_{x}+2 G \rho^{-1} G_{x}=-f|G| G \rho^{-1},
\end{aligned}
$$

$$
\begin{aligned}
(x, t) & \in[0, L] \times[0, T], \\
\rho(x, 0)=\rho_{0}(x), \quad G(x, 0)=G_{0}(x), & x \in[0, L], \\
G(0, t)=g_{0}(t), \quad G(L, t)=g_{1}(t), & t \in[0, T],
\end{aligned}
$$

where $\rho$ is mass density, $G$ is momentum density (averaged through the pipe crosssection), $\sigma=\sigma(\rho)$ is the isothermal speed of sound, $L$ is pipe length, and $f=f(|G|)$ $>0$ is a friction factor. We assume that the friction factor is described by the Moody diagram; see [12, pp. 288-289]. In this case there exist positive constants $G_{c}$ and $f_{0}$ such that $f(|G|)=f_{0}|G|^{-1}$ for $|G| \leqslant G_{c}$. There also exists a positive constant $f_{1}$ such that

$$
\lim _{|G| \rightarrow \infty} f(|G|)=f_{1}
$$

The boundary conditions above correspond to supplying the mass rate of flow at $x=$ $0, L$. Conditions on the data and the friction factor guaranteeing the existence of global smooth solutions to (1.3) have been given by the author in [7].

We now describe the finite element spaces used for our procedure for (1.1). Set $I=[0,1]$ and for $E \subset I$ define

$$
P_{k}(E)=\left\{z: I \rightarrow \mathbf{R}|z|_{E} \text { is a polynomial of degree } \leqslant k\right\} .
$$

For the partition $\delta=\left\{0=x_{0}<x_{1}<\cdots<x_{N}=1\right\}$, we define $I_{i}=\left[x_{i-1}, x_{i}\right], h_{i}$ $=x_{i}-x_{i-1}$, and $h=\max h_{i}$. Set

$$
M_{k}(r, \delta)=\left\{z \in C^{k}(I) \mid z \in P_{r}\left(I_{i}\right), i=1,2, \ldots, N\right\}
$$

We shall often write $M$ for $M_{k}(r, \delta)$. We also set

$$
\frac{d}{d x} M=\frac{d}{d x} M_{k}(r, \delta)=\left\{\frac{d z}{d x} \mid z \in M_{k}(r, \delta)\right\} .
$$

We shall assume that the families of spaces $M_{k}(r, \delta)$ considered in this paper are based on meshes $\delta$ that are quasi-uniform, i.e., there exists $C_{1}>0$ independent of $h$ such that

$$
\min \frac{h_{i}}{h} \geqslant C_{1}
$$

If $f, g \in L^{2}(I)$, denote

$$
\langle f, g\rangle=\int_{0}^{1} f g d x .
$$

We propose the following method to approximate (1.1): 
Find $U:[0, T] \rightarrow M$ and $V:[0, T] \rightarrow d M / d x$ such that (suppressing explicit dependence on $x, t$ )

$$
\begin{aligned}
& \left\langle U_{t}, \chi\right\rangle-\left\langle a_{12} V, \chi_{x}\right\rangle+a_{12}(1, t) g_{1}(t) \chi(1) \\
& -a_{12}(0, t) g_{0}(t) \chi(0)=\left\langle f_{1}(U, V), \chi\right\rangle, \quad \chi \in M, \\
& \left\langle V_{t}+a_{21}(U, V) U_{x}+a_{22}(U, V) V_{x}, \chi\right\rangle \\
& +\beta\left[V(1, t)-g_{1}(t)\right] \chi(1)+\beta\left[V(0, t)-g_{0}(t)\right] \chi(0) \\
& =\left\langle f_{2}(U, V), \chi\right\rangle, \quad \chi \in \frac{d}{d x} M, \\
& U(0) \approx u_{0}, \quad V(0) \approx v_{0} .
\end{aligned}
$$

The terms multiplied by the constant $\beta$ in (1.6b) represent penalty terms to impose the boundary conditions. Let

$$
\gamma=\max _{(x, t) \in[0,1] \times[0, T]}\left|a_{22}(x, t, u(x, t), v(x, t))\right| .
$$

We show that the scheme (1.6) is convergent if $\beta \geqslant \gamma / 2$. If $a_{22} \equiv 0$, it follows that the scheme (1.6) is convergent for $\beta \equiv 0$. We must also require that $M \subset C^{1}(I)$, i.e., $k \geqslant 1$, if $a_{22} \neq 0$. Otherwise the term

$$
\left\langle a_{22}(U, V) V_{x}, \chi\right\rangle, \quad \chi \in \frac{d}{d x} M,
$$

will not be defined.

We denote by $H^{j}$, for $j$ a positive integer, the Sobolev space of functions on $I$ with $j$ derivatives in $L^{2}(I)$ and norm

$$
\|z\|_{j}^{2}=\sum_{k=0}^{j} \int_{0}^{1} \frac{d^{k} z}{d x^{k}} \frac{d^{k} z}{d x^{k}} d x, \quad\|z\|^{2}=\|z\|_{0}^{2} .
$$

We shall prove the following theorem in Section 5:

THEOREM 1. Let $(u, v)$ be the solution to (1.1) and assume that there exists $C_{2}<\infty$ such that

$$
\sup _{0 \leqslant t \leqslant T}\left(\|u\|_{r}+\|v\|_{r}\right) \leqslant C_{2}, \quad \sqrt{\int_{0}^{T}\left[\left\|u_{t}\right\|_{r}^{2}+\left\|v_{t}\right\|_{r}^{2}\right] d t} \leqslant C_{2} .
$$

Assume that $U(0) \in M, V(0) \in d M / d x$ satisfy, for some $C_{3}<\infty$,

$$
\left\|U(0)-u_{0}\right\|+\left\|V(0)-v_{0}\right\| \leqslant C_{3} h^{r} .
$$

Suppose that $\beta \geqslant \gamma / 2$, that $k \geqslant 1$ if $a_{22} \neq 0$, and that $r \geqslant 2$ if the system (1.1) is nonlinear. Then there exists $h_{0}>0$ and $C$ such that the solution $(U, V)$ of $(1.6)$ exists on $[0, T]$ for $h \leqslant h_{0}$ and such that

$$
\|U(t)-u(t)\|+\|V(t)-v(t)\| \leqslant C h^{r} \quad \text { for } t \in[0, T], h \leqslant h_{0} .
$$


We note that the estimate (1.9) is of optimal order for $v$. It has not yet been determined whether the order of this estimate for the approximation of $u$ can be improved in general. The optimality of the result (1.9) is discussed in more detail in Section 5 .

Our procedure should be compared to the standard finite element procedure [2], to be discussed in Section 2, for approximating the solution of first-order hyperbolic systems. Although high convergence rates can be proven for both the standard finite element procedure and our alternative procedure, there are important qualitative differences in the solutions they produce. It is shown in Section 2 that the numerical solution produced by the standard finite element procedure has dispersion properties unlike those of the exact solution of the differential equations. This behavior has been noted by, among others, Hedstrom [5] and Platzman [9]. We show in Section 2 that for a model problem which is a linearization of (1.3) our proposed procedure yields a solution with dispersion properties similar to those of the solution of the differential equations.

In Section 3 we discuss the qualitative nature of the solution of (1.3). We show that for short pipes ( $L$ small) or rough disturbances the dissipative term $-f|G| G \rho^{-1}$ is relatively unimportant and the solution to (1.3) is approximated by the solution to a wave equation. However, for long pipes and mild disturbances the dissipative term $-f|G| G \rho^{-1}$ is shown to be very important and the solution to (1.3) is approximated by the solution to a diffusion equation. Our numerical solution is shown to be close to that of the solution of the standard finite element method for the wave equation or the diffusion equation under the appropriate conditions.

Section 4 gives a result which indicates that accurate long-time integration of systems such as (1.3) is possible with our procedure. We note that accurate long-time integration of (1.3) is not possible with the standard finite element method due to the accumulation of round-off and truncation error.

2. Dispersion Analysis of an Example. Consider the hyperbolic system

$$
\begin{aligned}
& u_{t}+v_{x}=0, \quad(x, t) \in[0,1] \times[0, T], \\
& v_{t}+u_{x}=0, \quad(x, t) \in[0,1] \times[0, T], \\
& v(0, t)=v(1, t)=0, \quad t \in[0, T], \\
& u(x, 0)=u_{0}(x), \quad v(x, 0)=v_{0}(x), \quad x \in[0,1] .
\end{aligned}
$$

The solution of (2.1) is easily constructed through Fourier analysis. If

$$
u_{0}(x)=\sum_{0}^{\infty} a_{n} \cos n \pi x, \quad v_{0}(x)=\sum_{1}^{\infty} b_{n} \sin n \pi x,
$$

then

$$
u(x, t)=\sum_{0}^{\infty} A_{n} \cos \left(n \pi t+\theta_{n}\right) \cos n \pi x,
$$




$$
v(x, t)=\sum_{1}^{\infty} A_{n} \sin \left(n \pi t+\theta_{n}\right) \sin n \pi x,
$$

where $A_{0}=a_{0}, \theta_{0}=0, A_{n} \cos \theta_{n}=a_{n}$, and $A_{n} \sin \theta_{n}=b_{n}$.

Now consider two approximation procedures for (2.1). Let

$$
H_{0}^{1}=\left\{z \in H^{1} \mid z(0)=z(1)=0\right\}, \quad M^{0}=M_{k}(r, \delta) \cap H_{0}^{1},
$$

and define the standard finite element approximation [2] to be the functions $U$ :

$[0, T] \rightarrow M$ and $V:[0, T] \rightarrow M^{0}$ such that

$$
\begin{gathered}
\left\langle U_{t}, W\right\rangle+\left\langle V_{x}, W\right\rangle=0, \quad W \in M, \\
\left\langle V_{t}, W\right\rangle+\left\langle U_{x}, W\right\rangle=0, \quad W \in M^{0}, \\
U(0) \approx u_{0}, \quad V(0) \approx v_{0} .
\end{gathered}
$$

It is instructive to study an explicit solution of (2.2) in the case $k=0, r=1$, and $\delta$ is a uniform partition. For $N$ a positive integer, set $h=1 / N$ and $x_{i}=i h, i=$ $0, \ldots, N$. Define the interpolation operator $P: C(I) \rightarrow M=M_{0}(1, \delta)$ by the relations

$$
\operatorname{Pz}\left(x_{i}\right)=z\left(x_{i}\right) \text { for } i=0, \ldots, N
$$

If

$$
U(0)=\sum_{0}^{N} a_{n} P(\cos n \pi x), \quad V(0)=\sum_{1}^{N} b_{n} P(\sin n \pi x),
$$

then

$$
\begin{aligned}
& U(t)=\sum_{0}^{N} A_{n} \cos \left(\Gamma_{n} t+\theta_{n}\right) P(\cos n \pi x), \\
& V(t)=\sum_{1}^{N} A_{n} \sin \left(\Gamma_{n} t+\theta_{n}\right) P(\sin n \pi x),
\end{aligned}
$$

where $A_{0}=a_{0}, A_{n} \cos \theta_{n}=a_{n}, A_{n} \sin \theta_{n}=b_{n}$, for $n=1, \ldots, N$, and

$$
\Gamma_{n}=\frac{3 \sin n h \pi}{h(2+\cos n h \pi)}
$$

Note that $\Gamma_{N}=0$. Thus, we see that

$$
U(t)=P(\cos N \pi x), \quad V(t)=P(\sin N \pi x)=0
$$

is a nonconstant steady-state solution to (2.2). Furthermore, we see from Graph \#1 that the most spatially oscillatory components of the solution of (2.2) have a low frequency in time even though the spatially oscillatory components of the solution of (2.1) have high frequency in time.

We can also see this phenomena for (2.2) and general spaces $M=M_{k}(r, \delta)$ as 
follows. Since

$$
\operatorname{dim} \frac{d}{d x} M^{0}+1<\operatorname{dim} M
$$

there exists a nonconstant $\hat{z} \in M$ such that

$$
\left\langle\chi_{x}, \hat{z}\right\rangle=-\left\langle\chi, \hat{z}_{x}\right\rangle=0, \quad \chi \in M^{0} .
$$

Hence

$$
U(t)=\hat{z}, \quad V(t)=0
$$

is a nonconstant steady-state solution of (2.2). We note by (2.5) that $\hat{z}$ must be a highly spatially oscillatory function.

Our new procedure to solve $(2.1)$ is the following. Find $U:[0, T] \rightarrow M=$ $M_{k}(r, \delta)$ and $V:[0, T] \rightarrow d M / d x$ such that

$$
\begin{gathered}
\left\langle U_{t}, W\right\rangle-\left\langle V, w_{x}\right\rangle=0, \quad W \in M, \\
\left\langle V_{t}, W\right\rangle+\left\langle U_{x}, W\right\rangle=0, \quad W \in \frac{d}{d x} M, \\
U(0) \approx u_{0}, \quad V(0) \approx v_{0} .
\end{gathered}
$$

We note that by elimination of $V$ in (2.7) we see that $U$ satisfies

$$
\left\langle U_{t t}, W\right\rangle+\left\langle U_{x}, W_{x}\right\rangle=0, \quad W \in M .
$$

Hence, our procedure reduces in this special case to the standard method for the wave equation.

An explicit solution to (2.7) can be constructed as follows. Let $\lambda_{k}$ be the $k$ th positive Rayleigh-Ritz approximate eigenvalue with eigenfunction $U_{k} \in M$ for the problem

$$
-u^{\prime \prime}=\lambda u, \quad x \in[0,1], \quad u^{\prime}(0)=u^{\prime}(1)=0 .
$$

Thus, $0<\lambda_{1}<\lambda_{2}<\cdots\left\langle\lambda_{M}\right.$ and $\lambda_{k}\left\langle U_{k}, W\right\rangle=\left\langle U_{k_{x}}, W_{x}\right\rangle, W \in M$, where $M=$ $\operatorname{dim} M-1$. Let $\omega_{k}=\sqrt{\lambda_{k}}$. Also, set $\omega_{0}=0$ and $U_{0}=1$. If

$$
U(0)=\sum_{0}^{M} a_{k} U_{k}, \quad V(0)=\sum_{1}^{M} b_{k}\left(-\omega_{k}^{-1} U_{k}\right)_{x}
$$

then

$$
\begin{aligned}
& U(t)=\sum_{0}^{M} A_{k} \cos \left(\omega_{k} t+\theta_{k}\right) U_{k}, \\
& V(t)=\sum_{1}^{M} A_{k} \sin \left(\omega_{k} t+\theta_{k}\right)\left(-\omega_{k}^{-1} U_{k}\right)_{x},
\end{aligned}
$$

where $A_{0}=a_{0}, \theta_{0}=0, A_{k} \cos \theta_{k}=a_{k}$, and $A_{k} \sin \theta_{k}=b_{k}$. It is well known [11, p. 223] that $\omega_{k} \geqslant k \pi$ for $k=1, \ldots, M$. Hence, we do not obtain nonconstant steady states for (2.7), and states which are spatially oscillatory have high frequency in time. 
In the case $M=M_{0}(1, \delta)$, where $\delta$ is a uniform mesh of size $1 / N, N$ a positive integer, we can take $U_{k}=P(\cos k \pi x)$ and

$$
\omega_{k}=2 \sin \left(\frac{k \pi h}{2}\right)\left(1-\frac{2}{3} \sin ^{2} \frac{k \pi h}{2}\right)^{-1 / 2} h^{-1}
$$

A comparison of the graph of $\omega_{k}$ and $\Gamma_{k}$ with the graph of $\nu_{k}=k \pi$ (see Graph \#1) shows the superior dispersion relation given by scheme (2.7). Intuitively, the graph shows that only about one-half of the degrees of freedom in the standard method are useful in approximating the solution, whereas for the proposed method all of the degrees of freedom are useful.

\section{GraPH \#1 (from Platzman, [9])}

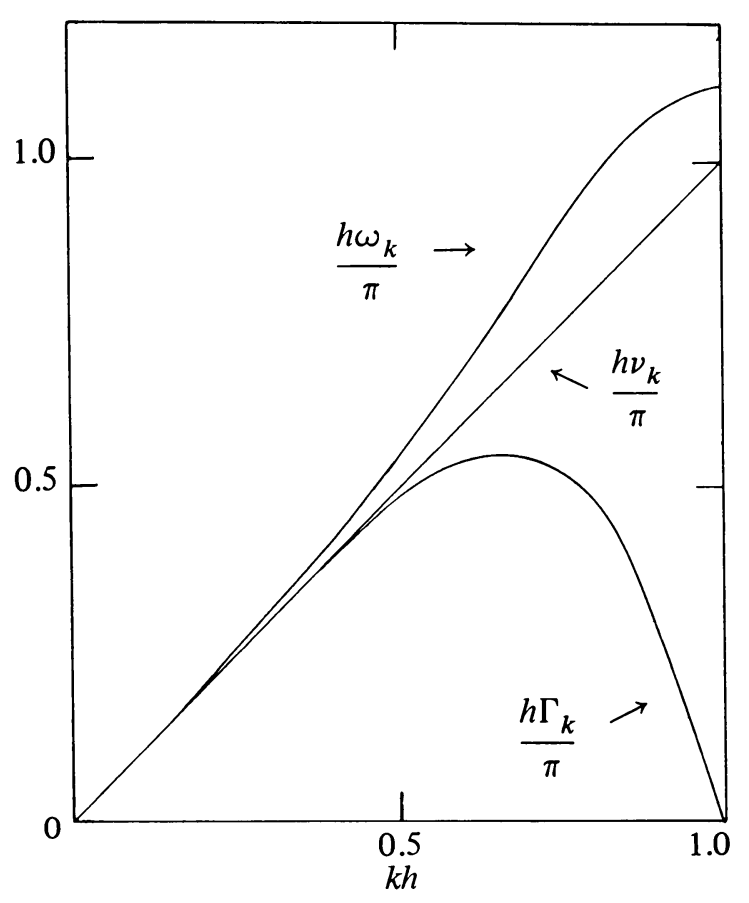

3. Application to the Modeling of Gas Flow in a Pipe. For high frequency disturbances, solutions of Eq. (1.3) behave very much like solutions of the usual second-order wave equation, and for low frequency disturbances they behave very much like solutions of a heat equation. In this section we indicate the sense in which this is true for a linearized version of (1.3) and examine the behavior of our numerical method in these two limiting situations.

If we linearize (1.3) about constant mass density $\hat{\rho}>0$ and momentum density $\hat{G}=0$, we obtain the system (after scaling the length)

$$
\begin{aligned}
& \rho_{t}+L^{-1} G_{x}=0, \quad(x, t) \in[0,1] \times[0, T], \\
& G_{t}+L^{-1} \hat{\sigma}^{2} \rho_{x}=-\hat{f} G,
\end{aligned}
$$$$
\rho(x, 0)=\rho_{0}(x), \quad G(x, 0)=G_{0}(x), \quad x \in[0,1],
$$ 
for positive constants $L, \hat{\sigma}$, and $\hat{f}$ where $L$ is pipe length, $\hat{\sigma}=\sigma(\hat{\rho})$ is the isothermal speed of sound, and $\hat{f}$ is a constant friction factor. We consider, for simplicity, the case of homogeneous boundary conditions

$$
G(0, t)=G(1, t)=0, \quad t \in[0, T] .
$$

We obtain, by eliminating the variable $G$ from (3.1)-(3.2), the damped wave equation

$$
\begin{aligned}
& \rho_{t t}-L^{-2} \hat{\sigma}^{2} \rho_{x x}=-\hat{f}_{t}, \quad(x, t) \in[0,1] \times[0, T], \\
& \rho(x, 0)=\rho_{0}(x), \quad \rho_{t}(x, 0)=-L^{-1} G_{0 x}(x), \quad x \in[0,1], \\
& \rho_{x}(0, t)=\rho_{x}(1, t)=0, \quad t \in[0, T] .
\end{aligned}
$$

Now suppose that the initial data is such that for $m$ a positive integer

$$
\rho(x, 0)=\alpha_{m} \cos (m \pi x) \text { and } \rho_{t}(x, 0)=\beta_{m} \cos (m \pi x) .
$$

Then it is easily checked that the solution to (3.5) is given by

$$
\rho(x, t)=\left[c_{m}^{+} e^{\gamma_{m}^{+} t}+c_{m}^{-} e^{\gamma_{m}^{-} t}\right] \cos (m \pi x)
$$

where

$$
\gamma_{m}^{ \pm}=-\frac{\hat{f}}{2}\left[1 \pm \sqrt{1-4 m^{2} \pi^{2} L^{-2} \hat{\sigma}^{2} \hat{f}^{-2}}\right], \quad c_{m}^{ \pm}=\frac{\beta_{m}-\gamma_{m}^{\mp} \alpha_{m}}{\gamma_{m}^{ \pm}-\gamma_{m}^{\mp}}
$$

If $\gamma_{m}^{-}=\gamma_{m}^{+}$for some $m$, then small modifications of the following arguments are necessary. Let

$$
\rho_{1}(x, t)=c_{m}^{+} e^{\gamma_{m}^{+} t} \cos (m \pi x), \quad \rho_{2}(x, t)=c_{m}^{-} e^{\gamma_{m}^{-}} \cos (m \pi x) .
$$

Note that $\rho=\rho_{1}+\rho_{2}$.

We first consider the case when

$$
m^{2} \pi^{2} L^{-2} \hat{\sigma}^{2} \hat{f}^{-2}>1
$$

A simple calculation shows that under the condition (3.7) we have that

$$
\left|\hat{f}_{i_{t}}\right|<<\left|L^{-2} \hat{\sigma}^{2} \rho_{i_{x x}}\right|,\left|\rho_{i_{t t}}\right|,
$$

for $(x, t) \in[0,1] \times[0, T], i=1,2$. (For functions $\phi, \psi \in C([0,1] \times[0, T])$ we say $|\phi|<<|\psi|$ for $(x, t) \in[0,1] \times[0, T]$ if and only if $|\phi| /|\psi|<<1$ for $(x, t)$ $\in[0,1] \times[0, T]$.) Hence, it follows that the solution to $(3.5)$ is approximated by the solution to the wave equation

$$
\begin{aligned}
& \rho_{t t}-L^{-2} \hat{\sigma}^{2} \rho_{x x}=0, \quad(x, t) \in[0,1] \times[0, T], \\
& \rho_{x}(0, t)=\rho_{x}(1, t)=0, \quad t \in[0, T], \\
& \rho(x, 0)=\alpha_{m} \cos (m \pi x), \quad \rho_{t}(x, 0)=\beta_{m} \cos (m \pi x), \quad x \in[0,1] .
\end{aligned}
$$


Next, we consider the case when

$$
m^{2} \pi^{2} L^{-2} \hat{\sigma}^{2} \hat{f}^{-2}<<1, \quad\left|\beta_{m}\right| \ll<\left|\alpha_{m} \hat{f}\right| .
$$

Then it is easily verified that

$$
\left|\rho_{2 t t}\right|<<\left|L^{-2} \hat{\sigma}^{2} \rho_{2 x x}\right|,\left|\hat{f} \rho_{2}\right| \quad \text { for }(x, t) \in[0,1] \times[0, T] .
$$

It also follows from (3.10) that $\left|c_{m}^{+}\right|<<\left|c_{m}^{-}\right|$so that

$$
\begin{aligned}
\left|\rho_{1_{t t}}\right|,\left|\hat{f}_{1_{t}}\right|,\left|L^{-2} \hat{\sigma}^{2} \rho_{1_{x x}}\right|<<\left|L^{-2} \hat{\sigma}^{2} \rho_{2_{x x}}\right|,\left|\hat{f} \rho_{2_{t}}\right| & \\
& \text { for }(x, t) \in[0,1] \times[0, T] .
\end{aligned}
$$

Hence, we see from (3.11) and (3.12) that under the conditions (3.10) we have that the solution of (3.5) is approximated by the solution of the diffusion equation

$$
\begin{aligned}
& -L^{-2} \hat{\sigma}^{2} \rho_{x x}=-\hat{f} \rho_{t}, \quad(x, t) \in[0,1] \times[0, T], \\
& \rho_{x}(0, t)=\rho_{x}(1, t)=0, \quad t \in[0, T], \\
& \rho(x, 0)=\alpha_{m} \cos (m \pi x), \quad x \in[0,1] .
\end{aligned}
$$

We shall now show that under the conditions (3.7) our numerical solution to (3.1) -(3.3) is close to the standard finite element method for the wave equation (3.9) and that under the conditions (3.10) our numerical solution to (3.1)-(3.3) is close to the standard finite element method for the diffusion equation (3.13).

Our finite element solution to $(3.1)-(3.3)$ is $\rho:[0, T] \rightarrow M, G:[0, T] \rightarrow$ $d M / d x$, such that

$$
\begin{aligned}
& \left\langle\rho_{t}, \chi\right\rangle-\left\langle L^{-1} G, \chi_{x}\right\rangle=0, \quad \chi \in M, \\
& \left\langle G_{t}, \chi\right\rangle+\left\langle L^{-1} \hat{\sigma}^{2} \rho_{x}, \chi\right\rangle=-\langle\hat{f} G, \chi\rangle, \quad \chi \in \frac{d}{d x} M, \\
& \rho(0) \approx \rho_{0}, \quad G(0) \approx G_{0} .
\end{aligned}
$$

We can eliminate $G$ from (3.14) to obtain the following finite element equation for $\rho:[0, T] \rightarrow M$

$$
\left\langle\rho_{t t}, \chi\right\rangle+\left\langle L^{-2} \hat{\sigma}^{2} \rho_{x}, \chi_{x}\right\rangle=-\left\langle\hat{f}_{t}, \chi\right\rangle, \quad \chi \in M,
$$

where $\rho(0)$ is as in $(3.14 \mathrm{c})$ and $\rho_{t}(0) \in M$ satisfies by $(3.14 \mathrm{a})$

$$
\left\langle\rho_{t}(0), \chi\right\rangle=\left\langle L^{-1} G(0), \chi_{x}\right\rangle, \quad \chi \in M .
$$

As in our discussion of the differential problem, we assume that

$$
\rho(0)=\alpha_{m} U_{m}, \quad \rho_{t}(0)=\beta_{m} U_{m}
$$

(we use here the notation for the eigenproblem (2.8) introduced in Section 2). The solution to $(3.15)$ is then given by

$$
\rho(t)=\left[c_{m}^{+} e^{\Gamma_{m}^{+} t}+c_{m}^{-} e^{\Gamma_{m}^{-} t}\right] U_{m},
$$


where

$$
\Gamma_{m}^{ \pm}=-\frac{\hat{f}}{2}\left[1 \pm \sqrt{1-4 \lambda_{k} L^{-2} \hat{\sigma}^{2} \hat{f}^{-2}}\right], \quad c_{m}^{ \pm}=\frac{\beta_{m}-\Gamma_{m}^{\mp} \alpha_{m}}{\Gamma_{m}^{ \pm}-\Gamma_{m}^{\mp}}
$$

An analysis similar to that given for the differential problem then establishes that if

$$
\lambda_{m} L^{-2} \hat{\sigma}^{2} \hat{f}^{-2} \gg>1
$$

we have that the solution to $(3.15)$ is approximated by the solution $\rho:[0, T] \rightarrow M$ to the standard finite element approximation for the wave equation

$$
\left\langle\rho_{t t}, \chi\right\rangle+\left\langle L^{-2} \hat{\sigma}^{2} \rho_{x}, \chi_{x}\right\rangle=0, \quad \chi \in M .
$$

Similarly, if

$$
\lambda_{m} L^{-2} \hat{\sigma}^{2} \hat{f}^{-2}<<1, \quad\left|\beta_{m}\right|<<\left|\alpha_{m} \hat{f}\right|,
$$

it can be verified that the solution to (3.15) is approximated by the solution $\rho$ :

$[0, T] \rightarrow M$ of the standard finite element approximation for the diffusion equation

$$
\left\langle L^{-2} \hat{\sigma}^{2} \rho_{x}, \chi_{x}\right\rangle=-\hat{f}\left\langle\rho_{t}, \chi\right\rangle, \quad \chi \in M, \quad \rho(0)=\alpha_{m} U_{m} .
$$

4. Long-Time Integration. We shall prove the following result:

Lemma 1. Let $(\rho, G)$ be the solution to the continuous problem (3.1)-(3.4).

There exist positive constants $\hat{C}$ and $D$ (depending on $\hat{\sigma}, L$, and $\hat{f}$, but independent of $T, \rho_{0}$, and $G_{0}$ ) such that

$$
\left\|\rho(t)-\int_{0}^{1} \rho_{0} d x\right\|+\|G(t)\| \leqslant \hat{C} e^{-D t}\left(\left\|\rho_{0}-\int_{0}^{1} \rho_{0} d x\right\|+\left\|G_{0}\right\|\right) .
$$

Also, if $\rho:[0, T] \rightarrow M$ and $G:[0, T] \rightarrow d M / d x$ is the numerical approximation of (3.1)-(3.4) defined by (3.14), then

$$
\left\|\rho(t)-\int_{0}^{1} \rho(0) d x\right\|+\|G(t)\| \leqslant \hat{C} e^{-D t}\left(\left\|\rho(0)-\int_{0}^{1} \rho(0) d x\right\|+\|G(0)\|\right) .
$$

Remark. This result implies that the dependence of the solution $\rho, G$ of (3.1)(3.4) at $t_{1}>t_{0}$ on $\rho_{0}, G_{0}$, and the boundary conditions for $t \in\left[0, t_{0}\right]$ decays exponentially (except for the dependence on $\int_{0}^{1} \rho\left(x, t_{0}\right) d x$, of course). It is essential that a numerical procedure for (3.1)-(3.4) have the property (4.2) if the accumulation of round-off error and truncation error is to be prevented from destroying the accuracy of the numerical solution after a finite interval of time.

Proof of Lemma 1. We shall prove the estimate (4.2) for the numerical solution given by (3.14). The proof that (4.1) holds for the differential problem is analogous. Suppose that the solution $\rho(t)=\Sigma_{0}^{M} c_{m}(t) U_{m} \in M$ to (3.14) has initial conditions

$$
\rho(0)=\sum_{0}^{M} \alpha_{m} U_{m}, \quad \rho_{t}(0)=\sum_{0}^{M} \beta_{m} U_{m}
$$


We assume in this section that the eigenfunctions $\left\{U_{m}\right\}$ have been normalized. Again, if $\gamma_{m}^{+}=\gamma_{m}^{-}$for some $m$, then small modifications of the following arguments are necessary.

It follows from (3.17) that

$$
c_{m}(t)=\left[c_{m}^{+} e^{\Gamma_{m}^{+} t}+c_{m}^{-} e^{\Gamma_{m}^{-} t}\right]
$$

By the orthonormality of the set $\left\{U_{m}\right\}$, we have that

$$
\left\|\rho(t)-\int_{0}^{1} \rho(0) d x\right\|^{2}=\sum_{1}^{M} c_{m}(t)^{2} .
$$

It follows from $(3.14 \mathrm{a})$ that

$$
G(t)=\sum_{1}^{M} c_{m}^{\prime}(t) L \lambda_{m}^{-1} U_{m_{x}}
$$

so

$$
\|G(t)\|^{2}=\sum_{1}^{M} c_{m}^{\prime}(t)^{2} L^{2} \lambda_{m}^{-1}
$$

It is easily checked that there exists a positive constant $C$, which is independent of $m$ and $\delta$, such that

$$
\left|c_{m}^{+}\right|^{2}+\left|c_{m}^{-}\right|^{2} \leqslant C\left[\alpha_{m}^{2}+\beta_{m}^{2} L^{2} \lambda_{m}^{-1}\right]
$$

We also note that since $\lambda_{m} \geqslant \lambda_{1} \geqslant \pi^{2}$ for all partitions $\delta$ and for $m=1, \ldots, M$ it follows that

$$
\operatorname{Re} \Gamma_{m}^{ \pm} \leqslant \operatorname{Re}\left\{-\frac{\hat{f}}{2}\left[1-\sqrt{1-4 \pi^{2} \hat{\sigma}^{2} L^{-2} \hat{f}^{-2}}\right]\right\} \equiv D
$$

for $m=1, \ldots, M$.

Hence, we have that

$$
c_{m}^{2}(t) \leqslant 2 e^{-2 D t}\left[\left|c_{m}^{+}\right|^{2}+\left|c_{m}^{-}\right|^{2}\right] \leqslant C e^{-2 D t}\left[\alpha_{m}^{2}+\beta_{m}^{2} L^{2} \lambda_{m}^{-1}\right]
$$

Since $c_{m}(0)=\alpha_{m}$ and $c_{m}^{\prime}(0)=\beta_{m}$, it follows from (4.3) and (4.5) that

$$
\sum_{1}^{M} \alpha_{m}^{2}=\left\|\rho(0)-\int_{0}^{1} \rho(0) d x\right\|^{2}, \quad \sum_{1}^{M} \beta_{m}^{2} L^{2} \lambda_{m}^{-1}=\|G(0)\|^{2} .
$$

Thus, if we sum (4.8) for $m=1, \ldots, M$, we obtain

$$
\left\|\rho(t)-\int_{0}^{1} \rho(0) d x\right\|^{2} \leqslant C e^{-2 D t}\left(\left\|\rho(0)-\int_{0}^{1} \rho(0) d x\right\|^{2}+\|G(0)\|^{2}\right) .
$$

Next, it is easy to show that there exists a positive constant $C$, which is independent of $m$ and $\delta$, such that

$$
\left|\left(\Gamma_{m}^{ \pm}\right)^{2} \lambda_{m}^{-1}\right| \leqslant C
$$


Hence, we have that

$$
\begin{aligned}
\|G(t)\|^{2}=\sum_{1}^{M} c_{m}^{\prime}(t)^{2} L^{2} \lambda_{m}^{-1} & =\sum_{1}^{M}\left(c_{m}^{+} \Gamma_{m}^{+} e^{\Gamma_{m}^{+} t}+c_{m}^{-} \Gamma_{m}^{-} e^{\Gamma_{m}^{t}}\right)^{2} L^{2} \lambda_{m}^{-1} \\
& \leqslant C e^{-2 D t} \sum_{1}^{M}\left[\left|c_{m}^{+}\right|^{2}+\left|c_{m}^{-}\right|^{2}\right] \\
& \leqslant C e^{-2 D t}\left(\left\|\rho(0)-\int_{0}^{1} \rho(0) d x\right\|^{2}+\|G(0)\|^{2}\right)
\end{aligned}
$$

We note that the estimate (4.2) is not valid for the standard finite element solution to (3.1)-(3.4). The standard finite element solution to (3.1)-(3.4) is obtained from (2.2) by replacing $U$ with $\rho$ and $V$ with $G$ and by adding $-\hat{f}\langle G, W\rangle$ to the right side of the second equation. Since (see (2.5))

$$
\rho(t) \equiv \hat{z}, \quad G(t) \equiv 0,
$$

is a nonconstant steady-state solution, it is clear that (4.2) cannot be verified for the standard finite element method.

5. Proof of Theorem 1. We shall fix $r>k \geqslant 1$ and derive error estimates for the scheme (1.6) for the family of spaces

$$
M=M_{k}(r, \delta)
$$

satisfying the quasi-uniformity assumption (1.5). We note in the statement of Theorem 1 that we may allow $k=0$ if $a_{22} \equiv 0$ and $r=1$ if (1.1) is linear. These special cases can be proven by a variant of the following argument.

In what follows we denote by $W^{j, \infty}$ the Sobolev space of functions with $j$ weak derivatives in $L^{\infty}(I)$ and norm

$$
|z|_{j}=\sum_{k=0}^{j}\left\|\frac{d^{k} z}{d x^{k}}\right\|_{L^{\infty}(I)}, \quad|z|=|z|_{0} .
$$

It is well known that there exists $C_{4}<\infty$, depending only on $C_{1}$ of (1.5) and $r$, such that the following inverse hypothesis holds for $\chi \in M$,

$$
h^{1 / 2}|\chi|+h \chi_{\chi}\left\|\leqslant C_{4}\right\| \chi\left\|, \quad h^{1 / 2}\left|\chi_{x}\right|+h \chi_{x x}\right\| \leqslant C_{4}\left\|\chi_{x}\right\| .
$$

It is also well known that the spaces $M$ satisfy the following approximation property:

There exists $C_{5}<\infty$ such that for $2 \leqslant s \leqslant r+1$ and $z \in H^{s}$,

$$
\inf _{\chi \in M}\left(\|z-\chi\|+h\left\|_{z}-\chi\right\|_{1}+h^{2}\left\|_{z}-\chi\right\|_{2}+h^{3 / 2}|z-\chi|_{1}\right) \leqslant C_{5} h^{s}\left\|_{z}\right\|_{s},
$$

and for $1 \leqslant s \leqslant r$ and $z \in H^{s}$,

$$
\inf _{\chi \in \frac{d}{d x} M}\left(\|z-\chi\|+h\left\|_{z}-\chi\right\|_{1}+h^{1 / 2}|z-\chi|_{0}\right) \leqslant C_{5} h^{s}\left\|_{z}\right\|_{s} .
$$


We also use the fact that there exists $C_{6}<\infty$ so that if $z \in H^{2}$ and $z_{x}(0)=z_{x}(1)$ $=0$, then we can choose $\chi \in M$ such that $\chi_{x}(0)=\chi_{x}(1)=0$ and

$$
\|z-\chi\|_{1}+h\left\|_{\chi}\right\|_{2} \leqslant C_{6} h\left\|_{z}\right\|_{2} .
$$

In what follows, $C$ will denote a constant which depends on the Lipschitz constants for $a_{i j}$ and $f_{i}, \alpha$, and $C_{1}$ through $C_{6}$, but which is independent of $\delta$. It will be allowed to vary from estimate to estimate. When the arguments for $a_{i j}, f_{i}$ are omitted we assume that the functions are evaluated at $(x, t, u(x, t), v(x, t))$.

We wish to define the weighted $L^{2}$ projection of $v, R=R(v) \in d M / d x$, by relations

$$
\left\langle a_{12}(v-R(v)), W\right\rangle=0, \quad W \in \frac{d}{d x} M
$$

It follows by the approximation property (5.3) and (1.2) that $\eta_{2}=v-R(v)$ satisfies

$$
\left\|\eta_{2}\right\| \leqslant C\left\|_{v}\right\|_{r} h^{r}
$$

It then follows by the approximation property (5.3) and the inverse hypothesis (5.1) that

$$
h\left\|\eta_{2}\right\|_{1}+h^{1 / 2}\left|\eta_{2}\right| \leqslant C h^{r}\left\|_{v}\right\|_{r}
$$

We obtain an estimate for $\eta_{2}$ by differentiating (5.5). We then obtain

$$
\left\langle a_{12} \eta_{2}, W\right\rangle+\left\langle\left(\frac{d}{d t} a_{12}\right) \eta_{2}, W\right\rangle=0, \quad W \in \frac{d}{d x} M
$$

it follows that

$$
\left\|\eta_{2_{t}}\right\| \leqslant C\left\|\eta_{2}\right\|+C \inf _{\chi \in \frac{d}{d x} M}\left\|v_{t}-\chi\right\| \leqslant C h^{r}\left(\|v\|_{r}+\left\|v_{t}\right\|_{r}\right) .
$$

As before, by (5.3) and (5.1) we can then obtain

$$
h\left\|_{\eta_{t}}\right\|_{1}+h^{1 / 2}\left|\eta_{2}\right| \leqslant C h^{r}\left(\|v\|_{r}+\left\|v_{t}\right\|_{r}\right) .
$$

We also wish to define the following approximation of $u, Q=Q(u, v) \in M$, by the relations

$$
\begin{gathered}
\left\langle a_{21}(u-Q(u, v))_{x}, \chi_{x}\right\rangle+\left\langle a_{22} \eta_{2}, \chi_{x}\right\rangle \\
+\beta \eta_{2}(1, t) \chi_{x}(1)+\beta \eta_{2}(0, t) \chi_{x}(0)=0, \quad \chi \in M, \\
\int_{0}^{1}(u-Q(u, v)) d x=0 .
\end{gathered}
$$

Set $\eta_{1}=u-Q(u, v)$. It follows from (5.1) and (5.7) that

$$
\begin{array}{ll}
\left|\eta_{2}(1, t) \chi_{x}(1)\right| \leqslant C h^{r-1}\left\|\chi_{x}\right\|\|v\|_{r}, & \chi \in M, \\
\left|\eta_{2}(0, t) \chi_{x}(0)\right| \leqslant C h^{r-1}\left\|\chi_{x}\right\|\|v\|_{r}, & \chi \in M .
\end{array}
$$


Hence, it follows that

$$
\left\|\eta_{1}\right\| \leqslant C\left\|_{\eta_{2}}\right\|_{1}+C h^{r-1}\|v\|_{r}+C \inf _{x \in M}\|u-\chi\|_{1} \leqslant C h^{r-1}\left(\|v\|_{r}+\|u\|_{r}\right) .
$$

We can derive an $L^{2}$ estimate for $\eta_{1}$ by a variant of a frequently used duality argument. Let $f \in L^{2}(I), \int_{0}^{1} f d x=0$, and determine $\phi$ by

$$
-\left(a_{21} \phi_{x}\right)_{x}=f, \quad x \in[0,1], \quad \phi_{x}(0)=\phi_{x}(1)=0, \quad \int_{0}^{1} \phi d x=0 .
$$

Note that

$$
\phi_{x}(x)=-\frac{1}{a_{21}} \int_{0}^{x} f(s) d x .
$$

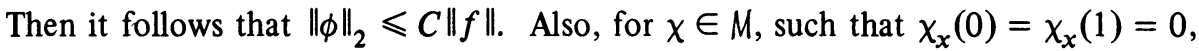
by (5.11)

$$
\begin{aligned}
\left\langle\eta_{1}, f\right\rangle & =\left\langle\eta_{1}-\left(a_{21} \phi_{x}\right)_{x}\right\rangle=\left\langle a_{21} \eta_{1 x}, \phi_{x}\right\rangle \\
& =\left\langle a_{21} \eta_{1_{x}},(\phi-\chi)_{x}\right\rangle+\left\langle a_{22} \eta_{2 x},(\phi-\chi)_{x}\right\rangle+\left\langle\eta_{2},\left(a_{22} \phi_{x}\right)_{x}\right\rangle
\end{aligned}
$$

Now, if we choose $\chi$ to approximate $\phi$ as in (5.4) so that $\|\phi-\chi\|_{1} \leqslant C h\|\phi\|_{2}$, it follows that

$$
\left|\left\langle\eta_{1}, f\right\rangle\right| \leqslant C\left[h\left\|_{\eta_{1}}\right\|_{1}+h\left\|\eta_{2}\right\|_{1}+\left\|\eta_{2}\right\|\right]\|f\|
$$

Hence, since $\int_{0}^{1} \eta_{1} d x=0$, we can conclude that

$$
\left\|\eta_{1}\right\| \leqslant C h^{r}\left[\|u\|_{r}+\|v\|_{r}\right] \text {. }
$$

We can derive an estimate for $\eta_{1}$ by differentiating (5.11) with respect to $t$ to obtain, as in the estimate for $\left\|\eta_{1}\right\|_{1}$,

$$
\left\|\eta_{1_{t}}\right\|_{1} \leqslant C h^{r-1}\left(\|u\|_{r}+\left\|u_{t}\right\|_{r}+\|v\|_{r}+\left\|v_{t}\right\|_{r}\right) \text {. }
$$

A duality argument similar to the previous such argument establishes that

$$
\left\|\eta_{1_{t}}\right\| \leqslant C h^{r}\left[\|u\|_{r}+\|v\|_{r}+\left\|u_{t}\right\|_{r}+\left\|v_{t}\right\|_{r}\right] .
$$

Set $\xi_{1}=U-Q(u, v), \xi_{2}=V-R(v)$. Then from (1.1), (1.6a), and (5.5) we see that

$$
\left\langle\xi_{1}, \chi\right\rangle-\left\langle a_{12} \xi_{2}, \chi_{x}\right\rangle=\left\langle\eta_{1}, \chi\right\rangle+\left\langle f_{1}(U, V)-f_{1}(u, v), \chi\right\rangle, \quad \chi \in M .
$$

It also follows from (1.1), (1.6b), and (5.11) that

$$
\begin{aligned}
\left\langle\xi_{2} t+a_{21}(u, v) \xi_{1 x}\right. & \left.+a_{22}(u, v) \xi_{2 x}, \chi\right\rangle+\beta \xi_{2}(1, t) \chi(1)+\beta \xi_{2}(0, t) \chi(0) \\
= & \left\langle\eta_{2}, \chi\right\rangle+\left\langle\left[a_{21}(u, v)-a_{21}(U, V)\right] U_{x}, \chi\right\rangle \\
& +\left\langle\left[a_{22}(u, v)-a_{22}(U, V)\right] V_{x}, \chi\right\rangle \\
& +\left\langle f_{2}(U, V)-f_{2}(u, v), \chi\right\rangle, \quad \chi \in \frac{d}{d x} M
\end{aligned}
$$


We prove the theorem by a variant of an argument used in [2] , [6], [10], [13] by showing that there exists a positive constant $C_{7}$ such that for any $t \in[0, T]$, if

$$
(U, V) \text { exists on }[0, t]
$$

such that

$$
\{(x, s, U(x, s), V(x, s)) \mid(x, s) \in[0,1] \times[0, t]\} \subseteq A,
$$

and

$$
\left|(u-U)_{x}(s)\right|+\left|(v-V)_{x}(s)\right| \leqslant 1 \text { for } 0 \leqslant s \leqslant t,
$$

then

$$
\|(u-U)(s)\|+\|(v-V)(s)\| \leqslant C_{7} h^{r}, \quad 0 \leqslant s \leqslant t .
$$

Let $B$ be a compact neighborhood of

$$
\{(x, s, u(x, s), v(x, s)) \mid(x, s) \in[0,1] \times[0, T]\}
$$

such that $B \subseteq$ interior of $A$.

It follows from the "inverse" hypothesis (5.1) that (5.22) implies the existence of a positive constant $C$ so that

$$
|(u-U)(s)|+|(v-V)(s)| \leqslant C h^{r-1 / 2}, \quad 0 \leqslant s \leqslant t .
$$

Hence, there exists a positive constant $h_{1}$ such that if $h \in\left(0, h_{1}\right]$, then (5.22) guarantees that

$$
\{(x, s, U(x, s), V(x, s)) \mid(x, s) \in[0,1] \times[0, t]\} \subseteq B .
$$

Similarly, it follows from the "inverse" hypothesis (5.1) that (5.22) implies the existence of a positive constant $C$ such that

$$
\left|(u-U)_{x}(s)\right|+\left|(v-V)_{x}(s)\right| \leqslant C h^{r-3 / 2}, \quad 0 \leqslant s \leqslant t .
$$

Hence, since $r \geqslant 2$, there exists a positive constant $h_{2}$ such that if $h \in\left(0, h_{2}\right]$, then (5.22) guarantees that

$$
\left|(u-U)_{x}(s)\right|+\left|(v-V)_{x}(s)\right| \leqslant 1 / 2 \quad \text { for } 0 \leqslant s \leqslant t .
$$

Now, $U(x, t), V(x, t),\left|(u-U)_{x}(t)\right|$, and $\left|(v-V)_{x}(t)\right|$ are continuous functions of $t$. Also, by the assumption (1.8) on the choice of $U(x, 0), V(x, 0)$ and the above discussion it follows that for $h \leqslant \min \left(h_{1}, h_{2}\right)$ we have (assuming without loss of generality that $C_{7} \leqslant C_{3}$ )

$$
\{(x, 0, U(x, 0), V(x, 0)) \mid x \in I\} \subseteq B \quad \text { and } \quad\left|(u-U)_{x}(0)\right|+\left|(v-V)_{x}(0)\right| \leqslant 1 / 2 .
$$

Suppose we have proven that (5.21) implies (5.22). We can thus conclude from the existence theory for ordinary differential equations that if $h \leqslant h_{0} \equiv$ $\min \left(h_{1}, h_{2}\right)$, then $(U, V)$ exists on $[0, T]$, 


$$
\begin{gathered}
\{(x, s, U(x, s), V(x, s)) \mid(x, s) \in[0,1] \times[0, T]\} \subseteq B, \\
\left|(u-U)_{x}(s)\right|+\left|(v-V)_{x}(s)\right| \leqslant 1 / 2, \quad 0 \leqslant s \leqslant T,
\end{gathered}
$$

and

$$
\|(u-U)(s)\|+\|(v-V)(s)\| \leqslant C_{7} h^{r}, \quad 0 \leqslant s \leqslant T .
$$

So, we assume that $(5.21)$ is valid. We shall proceed to prove the estimate (5.22). Since

$$
\{(x, s, U(x, s), V(x, s)) \mid(x, s) \in[0,1] \times[0, t]\} \subseteq A,
$$

we can assume (1.2) and the Lipschitz bounds on the coefficients where needed. We also note that it follows from (5.21) and Sobolev's inequality that

$$
\begin{aligned}
\left|U_{x}(s)\right| & +\left|V_{x}(s)\right| \leqslant\left|u_{x}(s)\right|+\left|v_{x}(s)\right|+1 \\
& \leqslant C\left\{\|u(s)\|_{2}+\|v(s)\|_{2}\right\}+1 \leqslant C C_{2}+1,
\end{aligned}
$$

for $0 \leqslant s \leqslant t$.

We set $\overline{a \xi_{1}} \in M$ to be the $L^{2}$ projection of $a_{21} \xi_{1} / a_{12}$ onto $M$, i.e.,

$$
\left\langle\frac{a_{21}}{a_{12}} \xi_{1}, \chi\right\rangle=\left\langle\overline{a \xi_{1}}, \chi\right\rangle, \quad \chi \in M .
$$

We use the following variant of a lemma of Dupont and Wahlbin [4] to estimate the error $\overline{a \xi_{1}}-a_{21} \xi_{1} / a_{12}$.

LEMMA 2. There is a constant $C$ such that if $b$ is a continuously differentiable function on $[0,1], \phi \in M$, and $\psi$ is the $L^{2}$ projection of $b \phi$ onto $M$, then

$$
\left\|(b \phi-\psi)_{x}\right\| \leqslant C\left|b_{x}\right|\|\phi\| .
$$

Proof of Lemma. For $\chi \in M$, we have by (5.1)

$$
\begin{aligned}
\left\|(b \phi-\psi)_{x}\right\| & \leqslant\left\|(b \phi-\chi)_{x}\right\|+\left\|(\chi-\psi)_{x}\right\| \\
& \leqslant\left\|(b \phi-\chi)_{x}\right\|+C h^{-1}\|\chi-\psi\| \\
& \leqslant\left\|(b \phi-\chi)_{x}\right\|+C h^{-1}(\|b \phi-\chi\|+\|b \phi-\psi\|) .
\end{aligned}
$$

It follows directly from [4] that $\|b \phi-\psi\| \leqslant C h\left|b_{x}\right|\|\phi\|$. The argument in [4] also shows that one can construct $\chi \in M_{r-1}(r, \delta)$ such that $\|b \phi-\chi\|+h\left\|(b \phi-\chi)_{x}\right\| \leqslant$ $C h\left|b_{x}\right|\|\phi\|$. The result follows by substituting the above two estimates in (5.25). Q.E.D.

We apply the lemma to obtain

$$
\left\|\overline{\left(a \xi_{1}\right)_{x}}-\left(\frac{a_{21}}{a_{12}} \xi_{1}\right)_{x}\right\| \leqslant C\left|\left(\frac{a_{21}}{a_{12}}\right)_{x}\right|\left\|\xi_{1}\right\| .
$$


We note that from (1.2) it follows that there exists a constant $C>1$ such that

$$
C^{-1}\left\|\xi_{1}\right\| \leqslant\left\|\frac{a_{21}}{a_{12}} \xi_{1}\right\| \leqslant C\left\|\xi_{1}\right\| .
$$

We now take $\chi=\overline{a \xi_{1}} \in M$ as our test function in (5.19). We obtain, since $\xi_{1_{t}}$ $\in M$, that

$$
\left\langle\xi_{1}, \overline{a \xi_{1}}\right\rangle=\left\langle\xi_{1}, \frac{a_{21}}{a_{12}} \xi_{1}\right\rangle
$$

Also,

$$
\left\langle\xi_{1}, \frac{a_{21}}{a_{12}} \xi_{1}\right\rangle=\frac{1}{2} \frac{d}{d t}\left\langle\frac{a_{21}}{a_{12}} \xi_{1}, \xi_{1}\right\rangle-\frac{1}{2}\left\langle\xi_{1},\left(\frac{d}{d t} \frac{a_{21}}{a_{12}}\right) \xi_{1}\right\rangle
$$

and by (5.26)

$$
\begin{aligned}
\left\langle a_{12} \xi_{2}, \overline{\left(a \xi_{1}\right)_{x}}\right\rangle & =\left\langle a_{12} \xi_{2},\left(\frac{a_{21}}{a_{12}} \xi_{1}\right)_{x}\right\rangle+O\left(\left\|\xi_{2}\right\|^{2}+\left\|\xi_{1}\right\|^{2}\right) \\
& =\left\langle a_{12} \xi_{2}, \xi_{1 x}\right\rangle+O\left(\left\|\xi_{2}\right\|^{2}+\left\|\xi_{1}\right\|^{2}\right) .
\end{aligned}
$$

Hence, we can obtain from (5.19), (5.27) and (5.6), (5.16), and (5.18) that

$$
\frac{1}{2} \frac{d}{d t}\left\langle\xi_{1}, \frac{a_{21}}{a_{12}} \xi_{1}\right\rangle-\left\langle a_{21} \xi_{2}, \xi_{1 x^{\prime}}\right\rangle \leqslant C\left[\left\|\xi_{1}\right\|^{2}+\left\|\xi_{2}\right\|^{2}\right]+C h^{2 r} .
$$

If we set $\chi=\xi_{2} \in d M / d x$ in (5.20) and use (5.6), (5.9), (5.16) and (5.23), we obtain

$$
\begin{aligned}
&\left\langle\xi_{2}, \xi_{2}\right\rangle+\left\langle a_{21} \xi_{1}, \xi_{2}\right\rangle+\left\langle a_{22} \xi_{2 x}, \xi_{2}\right\rangle+\beta \xi_{2}(1, s)^{2}+\beta \xi_{2}(0, s)^{2} \\
& \leqslant C h^{2 r}+C\left[\left\|\xi_{1}\right\|^{2}+\left\|\xi_{2}\right\|^{2}\right] .
\end{aligned}
$$

However,

$$
\begin{aligned}
\left\langle a_{22} \xi_{2 x}, \xi_{2}\right\rangle & =\frac{1}{2} \int_{0}^{1}\left(a_{22} \xi_{2}^{2}\right)_{x} d x-\frac{1}{2}\left\langle\left(a_{22}\right)_{x} \xi_{2}, \xi_{2}\right\rangle \\
& \leqslant \frac{1}{2} \gamma\left(\xi_{2}(1, s)^{2}+\xi_{2}(0, s)^{2}\right)+C\left\|\xi_{2}\right\|^{2}
\end{aligned}
$$

Thus, if we add (5.29) to (5.30) and use (5.31), we obtain

$$
\begin{aligned}
\frac{1}{2} \frac{d}{d t}\left[\left\langle\xi_{1}, \frac{a_{21}}{a_{12}} \xi_{1}\right\rangle\right. & \left.+\left\|\xi_{2}\right\|^{2}\right]+\left(\beta-\frac{1}{2} \gamma\right)\left(\xi_{2}(1, s)^{2}+\xi_{2}(0, s)^{2}\right) \\
& \leqslant C\left[\left\|\xi_{1}\right\|^{2}+\left\|\xi_{2}\right\|^{2}\right]+C h^{2 r} .
\end{aligned}
$$

We now obtain from Gronwall's lemma the result

$$
\begin{aligned}
\left\|\xi_{1}(s)\right\|^{2}+\left\|\xi_{2}(s)\right\|^{2} & +\left(\beta-\frac{1}{2} \gamma\right) \int_{0}^{r}\left[\xi_{2}(1, \sigma)^{2}+\xi_{2}(0, \sigma)^{2}\right] d \sigma \\
& \leqslant C h^{2 r} \text { for } 0 \leqslant s \leqslant t .
\end{aligned}
$$

Since it follows from (5.6) and (5.16) that $\left\|\eta_{1}\right\|^{2}+\left\|\eta_{2}\right\|^{2} \leqslant C h^{2 r}$, we have shown 
that

$$
\|(u-U)(s)\|+\|(v-V)(s)\| \leqslant C h^{r}, \quad 0 \leqslant s \leqslant t .
$$

Thus, we have shown that (5.21) implies (5.22). Hence, we can conclude (as our theorem asserts) that there exists $h_{0}>0$ such that if $0<h \leqslant h_{0}$, then $(U, V)$ exist on $[0, T]$ and

$$
\|(u-U)(t)\|+\|(v-V)(t)\| \leqslant C h^{r} \text { for } 0 \leqslant t \leqslant T .
$$

Q.E.D.

We also note that the following estimate is valid [1]:

$$
\left|\eta_{2}\right| \leqslant C h^{r}|v|_{r}
$$

We can use this estimate to conclude from (5.33) the following corollary.

COROLLARY 1. If in addition to the conditions of Theorem 1 we have that

$$
\int_{0}^{T}|v|_{r}^{2} d t<\infty
$$

and $\beta>\gamma / 2$, then the estimate

$$
\int_{0}^{T}\left[|v(1, t)-V(1, t)|^{2}+|v(0, t)-V(0, t)|^{2}\right] d t \leqslant C h^{2 r}
$$

is valid.

We now consider the optimality of the estimate (1.9). First, we consider the problem

$$
\begin{aligned}
& u_{t}+v_{x}=0, \quad(x, t) \in[0,1] \times[0, T], \\
& v_{t}+u_{x}=-\hat{f}, \\
& v(0, t)=g_{0}(t), \quad v(1, t)=g_{1}(t), \quad t \in[0, T], \\
& u(x, 0)=u_{0}(x), \quad v(x, 0)=v_{0}(x), \quad x \in[0,1],
\end{aligned}
$$

where $\hat{f}$ is a constant. The next lemma shows that if the solution $(u, v)$ to $(5.38)$ is smooth enough and if

$$
\left\|u_{0}-U(0)\right\| \leqslant C h^{r+1}, \quad\left\langle v_{0}-V(0), \chi\right\rangle=0, \quad \chi \in \frac{d}{d x} M,
$$

then the improved estimate

$$
h^{-1}\|u(t)-U(t)\|+\|v(t)-V(t)\| \leqslant C h^{r}, \quad t \in[0, T],
$$

can be obtained. We note that the estimate (5.40) is of optimal order in $h$ for both $u$ and $v$.

Lemma 3. Let $(u, v)$ be the solution to (5.38) and assume that there exists $C_{8}$ such that

$$
\sup _{0 \leqslant t \leqslant T}\left(\|u\|_{r+1}+\|v\|_{r}\right) \leqslant C_{8}, \quad \sqrt{\int_{0}^{T}\left\|u_{t}\right\|_{r+1}^{2} d t} \leqslant C_{8} .
$$


Let $(U, V)$ be the solution to (1.6) with $a_{12} \equiv 1, f_{1} \equiv 0, a_{21} \equiv 1, a_{22} \equiv 0, f_{2}(u, v)$ $\equiv-\hat{f}, \beta=0, k \geqslant 0$, and $r \geqslant 1$. Assume that $(U(0), V(0))$ satisfies (5.39). Then the estimate (5.40) is valid.

Proof. It is easily checked that (using the notation of Theorem 1)

$$
\left\langle\xi_{1}, \chi\right\rangle-\left\langle\xi_{2}, \chi_{x}\right\rangle=\left\langle\eta_{1}, \chi\right\rangle, \quad \chi \in M
$$

and

$$
\left\langle\xi_{2}, \chi\right\rangle+\left\langle\xi_{1}, \chi\right\rangle=-\hat{f}\left\langle\xi_{2}, \chi\right\rangle, \quad \chi \in \frac{d}{d x} M
$$

We set $\chi=\xi_{1}$ in (5.41), $\chi=\xi_{2}$ in (5.42), and add the resulting equations to obtain

$$
\frac{1}{2} \frac{d}{d t}\left[\left\|\xi_{1}\right\|^{2}+\left\|\xi_{2}\right\|^{2}\right] \leqslant \frac{1}{2}\left\|\eta_{1}\right\|^{2}+\frac{1}{2}\left\|\xi_{1}\right\|^{2}+|\hat{f}|\left\|\xi_{2}\right\|^{2} .
$$

Now it follows from standard estimates that in this case

$$
\left\|\eta_{1}\right\| \leqslant C h^{r+1}\|u\|_{r+1}, \quad\left\|\eta_{1}\right\| \leqslant C h^{r+1}\left\|u_{t}\right\|_{r+1}, \quad\left\|\eta_{2}\right\| \leqslant C h^{r}\left\|_{v}\right\|_{r} .
$$

Also, we can conclude from (5.39) and (5.44) that

$$
\left\|\xi_{1}(0)\right\| \leqslant C h^{r+1}, \quad \xi_{2}(0) \equiv 0 .
$$

Hence, it follows from (5.43), (5.44), (5.45), and Gronwall's inequality that

$$
\left\|\xi_{1}(t)\right\|^{2}+\left\|\xi_{2}(t)\right\|^{2} \leqslant C h^{2(r+1)}, \quad t \in[0, T]
$$

The result (5.40) now follows from (5.44) and (5.46). Q.E.D.

We conjecture that the estimate $(5.40)$ is valid in general if the solution $(u, v)$ to (1.1) is smooth enough, if $a_{22} \equiv 0$, and if

$$
\left\|u_{0}-U(0)\right\| \leqslant C h^{r+1}, \quad\left\langle a_{12}\left(v_{0}-V(0)\right), \chi\right\rangle=0, \quad \chi \in \frac{d}{d x} M .
$$

Next, consider the system

$$
\begin{aligned}
& u_{t}+v_{x}=f_{1}(x), \quad(x, t) \in[0,1] \times[0, T], \\
& v_{t}+u_{x}+v_{x}=f_{2}(x), \\
& v(0, t)=g_{0}(t), \quad v(1, t)=g_{1}(t), \quad t \in[0, T], \\
& u(x, 0)=u_{0}(x), \quad v(x, 0)=v_{0}(x), \quad x \in[0,1] .
\end{aligned}
$$

Let the space $M=M_{k}(r, \delta)$ where $\delta$ is a uniform partition with mesh length $h$, i.e., $h=1 / N$ for $N$ a positive integer and $x_{j}=j h$.

Set $\eta_{1}=u-Q(u, v)$ as before. Let $(U, V)$ be the solution of scheme (1.6) for (5.47) with $\beta=1$ and initial conditions

$$
V(0)=R\left(v_{0}\right), \quad U(0)=Q\left(u_{0}, v_{0}\right) .
$$

Let $M=M_{1}(2, \delta)$. It can be shown that if $(u, v)$ is a smooth solution to (5.47), 
then there exists a constant $C_{9}$ such that

$$
\left\|\eta_{1}\right\|+\left\|\eta_{1}\right\| \leqslant C_{9} h^{5 / 2}
$$

This result can be used in the analysis of Theorem 1 to show that if $(u, v)$ is a smooth solution to (5.47), then the result

$$
h^{-1 / 2}\|u(t)-U(t)\|+\|v(t)-V(t)\| \leqslant C h^{2}, \quad t \in[0, T],
$$

can be proven. The estimate (5.50) cannot be improved since it can be proven by techniques similar to those used in [3] that the estimate at $t=0$,

$$
h^{-1 / 2}\left\|u_{0}-Q\left(u_{0}, v_{0}\right)\right\|+\left\|_{v_{0}}-R\left(v_{0}\right)\right\| \leqslant C h^{2},
$$

cannot be improved.

It can also be shown that if $M=M_{2}(4, \delta)$ and if the initial conditions are determined by (5.48), then the estimate (1.9) cannot be improved.

Acknowledgements. The author wishes to thank Professors Todd Dupont, George Platzman, and Ridgway Scott for helpful advice on the writing of this paper.

Department of Mathematics

University of Michigan

Ann Arbor, Michigan 48109

1. J. DOUGLAS, JR., T. DUPONT \& L. WAHLBIN, “Optimal $L_{\infty}$ error estimates for Galerkin approximations to solutions of two-point boundary value problems," Math. Comp., v. 29, 1975, pp. $475-483$.

2. T. DUPONT, "Galerkin methods for modeling gas pipelines," Constructive and Computational Methods for Differential and Integral Equations, Lecture Notes in Math., Vol. 430, SpringerVerlag, Berlin and New York, 1974.

3. T. DUPONT, "Galerkin methods for first order hyperbolics: an example," SIAM J. Numer. Anal., v. 10, 1973, pp. 890-899.

4. T. DUPONT \& L. WAHLBIN, " $L 2$ optimality of weighted $H^{1}$ projections into piecewise polynomial spaces," Manuscript, Dept. of Math., Univ. of Chicago, 1974.

5. G. HEDSTROM, "The Galerkin method based on Hermite cubics," SIAM J. Numer. Anal., v. 16, 1979, pp. 385-393.

6. M. LEES, "A linear three level difference scheme for quasilinear parabolic equations,"

Math. Comp., v. 20, 1966, pp. 516-522.

7. M. LUSKIN, "On the existence of global smooth solutions for a model equation for fluid flow in a pipe," Manuscript, Dept. of Math., Ecole Polytechnique Fédérale de Lausanne, 1980.

8. M. LUSKIN, "A finite element method for first order hyperbolic systems in two space variables," Manuscript, Dept. of Math., Univ. of Michigan, 1978.

9. G. PLATZMAN, "Normal modes of the world ocean. Part 1. Design of a finite-element barotropic model," J. Phys. Oceanogr., v. 8, 1979, pp. 323-343.

10. H. RACHFORD, JR., "Two-level discrete-time Galerkin approximations for second order nonlinear parabolic partial differential equations," SIAM J. Numer. Anal., v. 10, 1973, pp. 1010-1026.

11. G. STRANG \& G. FIX, An Analysis of the Finite Element Method, Prentice-Hall, Englewood Cliffs, N.J., 1973.

12. V. STREETER, Fluid Mechanics, 5th ed., McGraw-Hill, New York, 1971.

13. L. WAHLBIN, "A dissipative Galerkin method applied to some quasilinear hyperbolic equations," R.A.I.R.O. Anal. Numer., v. 8, 1974, pp. 109-117. 